\title{
Effect of Narrowband Interference on Galileo E1 Signal Receiver Performance
}

\author{
Jie Zhang and Elena-Simona Lohan \\ Department of Communications Engineering, Tampere University of Technology, 33720 Tampere, Finland \\ Correspondence should be addressed to Jie Zhang, jie.zhang@tut.fi
}

Received 19 August 2011; Revised 9 November 2011; Accepted 13 December 2011

Academic Editor: Gyu-In Jee

Copyright ( 2011 J. Zhang and E.-S. Lohan. This is an open access article distributed under the Creative Commons Attribution License, which permits unrestricted use, distribution, and reproduction in any medium, provided the original work is properly cited.

Satellite navigation technology is becoming essential for civil application. The high-accuracy navigation service is demanded. However, the satellite signal may be exposed to the signal from other systems, which are sharing the same frequency band. This is a potential threat for the performance of navigation devices. The aim of this paper is to present an interference impact assessment in the context of global navigation based on the new modulation Composite Binary Offset Carrier (CBOC) that will be used for Galileo E1 civil signal. The focus is on the analysis of the Galileo CBOC-modulated signal robustness against narrowband interference.

\section{Introduction}

Satellite navigation is a process of providing autonomous global geospatial position with coverage all over the world. The navigation technology is essential for several civil applications, such as in the transportation field (e.g., road, rail, and aviation). Other applications, such as precision agriculture, wildlife behavior monitoring, surveying, and time-based applications are also based on the estimation of users' Position, Velocity, and Time (PVT) [1]. These applications, especially the ones dealing with safety, require high accuracy of users' PVT estimation.

The Global Navigation Satellite Systems (GNSSs) signals are allocated to Radio Navigation Satellite Services (RNSSs) and Aeronautical Radio Navigation Services (ARNSs) on a worldwide coprimary basis. However, the Global Navigation Satellite Systems (GNSSs) signals may be exposed to potential interference from other services that are sharing the similar frequency band. They could likely represent potential threats for GNSS devices. The interference may degrade the GNSS receivers' performance and compromise the safety.

Potential interferences are largely emanated from unintentional source or intentional jamming and spoofing of GNSS signal. Radio frequency interference (RFI) is one of the unintentional interference sources, whose frequency might be located in the satellite signal bands. RFI is normally classified as either wideband or narrowband, depending on whether its bandwidth is large or small relative to the bandwidth of the desired GNSS signal. Wideband interference can be a Gaussian waveform as in the case of UltraWideband (UWB) systems or harmonic from television transmission overcoming the front-end filter of a GNSS receiver [2]. Narrowband interference could originate from Amplitude Modulation (AM) or Frequency Modulation (FM) station.

The interference represents an impairing factor in GNSS application mainly due to the low power of the GNSS signal at the earth surface. The GNSS receiver may fail to acquire and track the satellite signals in the present of interference. Reports of measurement campaigns like [3] have shown that unaided GNSS receiver could experience loss of lock near FM and TV broadcast transmitter. Thus, it is important to have better understanding of the effects of RFI on GNSS receiver, in order to improve mitigation solutions.

The effect of narrowband interference has been assessed in the context of Global Positioning System (GPS) C/A signal in both theory and experiments [4-7]. However, the effect of narrowband interference on Galileo E1 signal, which is 
sharing the same frequency band with GPS C/A signal, has not been studied much. The code tracking performance in the presence of narrowband interference in Galileo E1 signal receiver may differ from that in the GPS signal due to different modulation used in Galileo E1 band $[8,9]$. Moreover, the modulation type of local replica used in a receiver could be different than that used in the transmitter $[10,11]$. Therefore, the theoretical equation developed by Betz [4] for evaluating the effect of narrowband interference on GPS C/A signal cannot be directly applied to the Galileo E1 signal. This is because it does not consider the aspect of receiver modulation type. The mathematical model needs to be modified in order to consider the modulation used in the receiver.

To summarize the above discussion, the effect of narrowband interference on Galileo E1 signal receiver, where the receiver type is also taken into account from both theory and simulations has not been evaluated. The goal of this paper is to present an analytical code tracking model of Galileo E1 signal in the presence of narrowband interference and evaluate the robustness of E1 signal towards the narrowband interference, taking into account the impact of different modulation types in the receiver. The code tracking accuracy of two tracking loops is compared: noncoherent earlyminus-late correlator (NELP) and coherent early-minus-late processing (CELP). The Cramér-Rao Lower Bound (CRLB) is used as reference in the delay tracking error studies. The results are also compared with simulations, which have been done with an open source Simulink simulator, Galileo E1 signal Tx-Rx chain built at Tampere University of Technology
[12]. The details of this simulator will be introduced in the following section.

The rest of the paper will be organized as follows: Section 2 presents the overview on the research of narrowband interference effects on GPS C/A signal. Section 3 compares the difference between Galileo E1 signal and GPS L1 signal and presents the modified analytical expression for Galileo E1 signal and numerical results, which takes the receiver modulation type into account. Section 4 summarizes the setup of the simulator. Simulation results are presented and discussed in Section 5, and finally, the conclusions are drawn in Section 6.

\section{Effect of Narrowband Interference on GPS Signal}

It is well known that a GNSS receiver can be exposed to many classes of undesired signals. Much research on effects of narrowband interference on GPS C/A signal has been conducted [4-6]. One of the most popular analytical models of GPS C/A signal code tracking in the presence of narrowband interference is introduced in $[4,6]$. This analysis uses complex baseband representations of signal and noise, and lowpass equivalent models of filtering and processing. From the mathematic point of view, it shows that the variance of the code tracking error (in chip) for CELP and equivalent expression for NELP are shown in (1) and (2) $[4,6]$,

$$
\begin{aligned}
\left(\sigma^{2}\right)_{\mathrm{CELP}}=B_{L}\left(1-0.5 B_{L} T\right) \times\left[\frac{\int_{-\beta_{r} / 2}^{\beta_{r} / 2} G_{W}(f) G_{S}(f) \sin ^{2}(\pi f \Delta) d f}{(2 \pi)^{2} C_{S}\left(\int_{-\beta_{r} / 2}^{\beta_{r} / 2} f G_{S}(f) \sin (\pi f \Delta) d f\right)^{2}}\right], \\
\left(\sigma^{2}\right)_{\mathrm{NELP}}=B_{L} T\left(1-0.5 B_{L} T\right) \times\left[\frac{\int_{-\beta_{r} / 2}^{\beta_{r} / 2} G_{W}(f) G_{S}(f) \sin ^{2}(\pi f \Delta) d f}{(2 \pi)^{2} C_{S}\left(\int_{-\beta_{r} / 2}^{\beta_{r} / 2} f G_{S}(f) \sin (\pi f \Delta) d f\right)^{2}}\right. \\
\\
\left.\quad+\frac{\left(\int_{-\beta_{r} / 2}^{\beta_{r} / 2} G_{W}(f) G_{S}(f) d f\right)^{2}-\left|\int_{-\beta_{r} / 2}^{\beta_{r} / 2} G_{W}(f) G_{S}(f) e^{i 2 \pi f \Delta} d f\right|}{4(2 \pi)^{2} T C_{S}^{2}\left(\int_{-\beta_{r} / 2}^{\beta_{r} / 2} f G_{S}(f) \sin (\pi f \Delta) d f \int_{-\beta_{r} / 2}^{\beta_{r} / 2} f G_{S}(f) \cos (\pi f \Delta) d f\right)^{2}}\right],
\end{aligned}
$$

where $G_{S}(f)$ : normalized GPS C/A BPSK signal spectrum $G_{S}(f)=T_{C} \operatorname{sinc}^{2}\left(\pi f T_{C}\right)$ with $\int_{-\infty}^{\infty} G_{S}(f)=1$, where $\operatorname{sinc}(y)=\sin (y) / y$ and $T_{C}$ is the chip period; $G_{W}(f)$ : $G_{W}(f)=N_{0}+C_{l} G_{l}(f) ; N_{0}$ is power density of the noise; $G_{l}(f)$ is normalized power spectrum density of interference with $\int_{-\infty}^{\infty} G_{l}(f)=1 ; C_{l}$ is the interference carrier power over infinite bandwidth; the noise and interference are each represented as zero mean, wide sense stationary stochastic process, independent of the signal, and statistically uncorrelated with each other; $C_{S}$ : power of the signal; $\beta_{r}$ : receiver front-end double-sided bandwidth; $\Delta$ : earlylate spacing in second; $B_{L}$ : one-sided equivalent rectangular bandwidth of the code tracking loop in $\mathrm{Hz}$; $T$ : Integration time in seconds used in the discriminator. 
The Cramér-Rao lower bound of time-of-arrival estimation for integration time $T$, then assuming the estimates are smoothed with the code tracking loop:

$$
\left(\sigma^{2}\right)_{\mathrm{LB}}=\frac{B_{L}\left(1-0.5 B_{L} T\right)}{(2 \pi)^{2} C_{S} \int_{-\beta_{r} / 2}^{\beta_{r} / 2} f^{2}\left(G_{S}(f) / G_{W}(f)\right) d f} .
$$

These theoretical expressions predict the performance of a GPS receiver in narrowband interference. They show that the interference near the carrier frequency has little effect on code tracking error, interference at a frequency midway between the carrier frequency, and first null has greater effect $[4]$.

\section{Effect of Narrowband Interference on Galileo Signal}

The equations above are derived for GPS L1 Binary Phase Shift Keying (BPSK) modulated signal. In order to check if above equations are applicable to Galileo E1 CBOCmodulated signal, we need to first have the knowledge of what is new in the CBOC modulation.

Galileo E1 signal and GPS L1 signal are allocated at the same carrier frequency. In order to minimize the interference between two signals, a new type of modulation, named "Multiplex Binary Offset Carrier (MBOC)" will be used on the Galileo E1 signal. The main idea behind MBOC is to minimize the interference with GPS L1 signal and to put a small amount of power on higher frequency, which could improve the code tracking performance $[8,9]$. In the latest Galileo Open Service Signal In Space Interface Control Document (OS SIS ICD) [13], Composite Binary Offset Carrier (CBOC) modulation, which is one of the implementation of MBOC is assigned for Galileo E1 band. This implementation is the sum (or difference) of two weighted Sine-Binary Offset Carrier (SinBOC) subcarrier waves [8]. The one used in E1 band is denoted via $\operatorname{CBOC}(6,1,1 / 11)$. It is the sum (or difference) of a $\operatorname{SinBOC}(1,1)$-modulated code and a SinBOC $(6,1)$-modulated code, which includes $1 / 11$ power from $\operatorname{Sin} B O C(6,1)$ component (and 10/11 power from $\operatorname{SinBOC}(1,1)$ component). Mathematically, the power spectral density (PSD) of $\operatorname{CBOC}(6,1,1 / 11)$ is

$$
G_{\mathrm{CBOC}(6,1,1 / 11)}(f)=\frac{10}{11} G_{\operatorname{SinBOC}(1,1)}(f)+\frac{1}{11} G_{\operatorname{SinBOC}(6,1)}(f),
$$

where $G_{\operatorname{SinBOC}(m, n)}(f)$ is the normalized PSD of a Sine$\operatorname{BOC}(m, n)$-modulation, given by $[10,14]$ :

$$
G_{\operatorname{SinBOC}(m, n)}(f)=\frac{1}{T_{c}}\left(\frac{\sin \left(\pi\left(T_{c} / N_{B}\right)\right) \sin \left(\pi f T_{c}\right)}{\pi f \cos \left(\pi\left(T_{c} / N_{B}\right)\right)}\right)^{2} .
$$

Here $T_{c}=1 / f c, f c$ is chip rate $(1.023 \mathrm{MHz}$ for E1 signal); the BOC modulation order $\left(N_{B}\right)$ is defined as $N_{B}=2 *(\mathrm{~m} / \mathrm{n})$. In time domain, $\operatorname{CBOC}(6,1,1 / 11)$ has several variables. The variables $\mathrm{CBOC}(+)$, which are the sum of two SinBOC signal, will be used in the data channel of E1 signal, and
CBOC $(-)$, where two SinBOC waves are subtracted, will be used in the pilot channel in E1 signal.

Normalized PSD of $\operatorname{CBOC}(6,1,1 / 11), \operatorname{SinBOC}(1,1)-$ and BPSK-modulated signals are shown in Figure 2. Compared with the PSD of BPSK, there are two main lobes located symmetrically around the center frequency in PSD of CBOC $(6,1,1 / 11)$ - and SinBOC $(1,1)$-modulated signals. The $\mathrm{CBOC}(6,1,1 / 11)$ has additional power at about $\pm 6 \mathrm{MHz}$ away from the center frequency.

As seen from (4), the transmitted E1 CBOC-modulated signal consists of two SinBOC signals, and more than $90 \%$ of signal power is from $\operatorname{SinBOC}(1,1)$ component. Therefore, the $\mathrm{CBOC}$ signal receiver could use either the $\mathrm{CBOC}$ modulated local replica in the receiver or $\operatorname{SinBOC}(1,1)$ modulated replica $[10,11,15]$. However, using difference modulation at the receiver side may cause the change on the signal power spectrum in the receiver. In order to check the impact of the modulation type in the receiver, we can model the transmitter-receiver chain for Galileo E1 signal as shown in Figure 1. The modulation at the transmitter is characterized by the transfer function $H_{\mathrm{Tx}}(f)$, which is the CBOC modulation transfer function. The Additive White Gaussian Noise and narrowband interference are then added in the transmitted signal. The front-end filter is used to limit the signal bandwidth, which is characterized by $B(f)$. After the front-end filter, the local replica $H_{\mathrm{Rx}}(f)$ at the receiver side is modulated with modulation whose transfer function is either $H_{\mathrm{CBOC}}(f)$ or $H_{\mathrm{BOC}}(f)$. The $G(f)$ is the signal part in the correlation between the received signal and modulated local replica. Depending on the modulation type used in the receiver, it can be expressed as:

$$
G(f)=C_{S}\left|H_{\mathrm{Tx}}(f) H_{\mathrm{Rx}} *(f)\right| .
$$

When the transmitter and receiver both have CBOC modulation type, (6) becomes

$$
G(f)=C_{S}\left|H_{\text {СвоС }}(f) H_{\text {СвОС }} *(f)\right| .
$$

When the receiver uses $\operatorname{Sin} \operatorname{BOC}(1,1)$-modulated local replica, the PSD will be:

$$
G(f)=C_{S}\left|H_{\mathrm{CBOC}}(f) H_{\mathrm{SinBOC}(1,1)} *(f)\right| .
$$

Above, $H_{\mathrm{CBOC}}(f)$ and $H_{\mathrm{BOC}}(f)$ are the transfer function of CBOC and SinBOC modulations as shown in (9) [10]:

$$
\begin{aligned}
& H_{\text {Свос }}(f) \\
& =e^{-j \pi f T_{C}} \frac{\sin \left(\pi f T_{C}\right)}{\pi f} \\
& \quad \times\left(w_{1} e^{-j \pi f\left(T_{C} / 2\right)} \tan \left(\frac{\pi f T_{C}}{2}\right)+a w_{2} \tan \left(\frac{\pi f T_{C}}{12}\right)\right), \\
& H_{\text {BOC }(1,1)}(f)=e^{-j \pi f\left(3 T_{C} / 2\right)} \frac{\sin \left(\pi f T_{C}\right)}{\pi f} \tan \left(\frac{\pi f T_{C}}{2}\right),
\end{aligned}
$$

where $T_{C}$ is chip rate; $w_{1}, w_{2}$ are weighting factor satisfying $w_{1}^{2}+w_{2}^{2}=1$ (e.g., $w_{1}=\sqrt{10 / 11}, w_{2}=\sqrt{1 / 11}$ for $\operatorname{CBOC}(6,1,1 / 11))$ and $a= \pm 1$ is a weight factor that used 


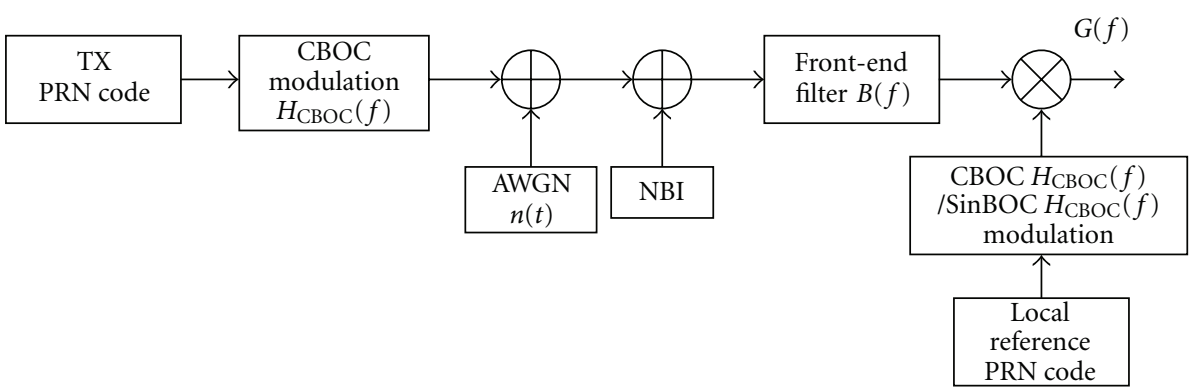

Figure 1: Block diagram of CBOC transmitter with reference CBOC- or SinBOC-modulated code at receiver.

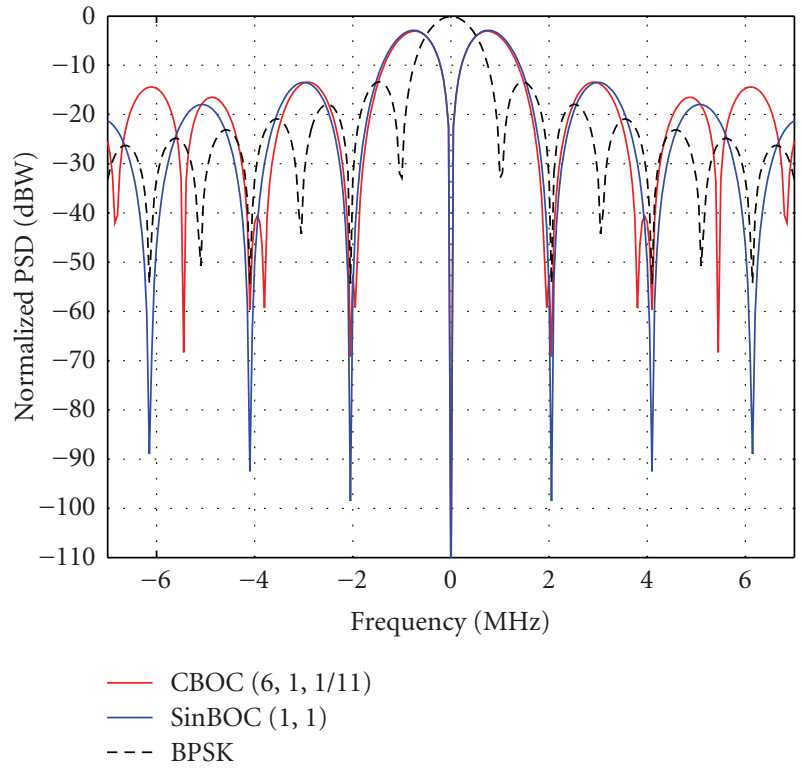

Figure 2: Normalized PSD of CBOC-, $\operatorname{SinBOC}(1,1)-$, and BPSKmodulated signal.

for data channels (for $\mathrm{CBOC}(+), a=1$ ) and pilot channel (for $\operatorname{CBOC}(-), a=-1$ ).

Usually the normalized PSDs are used [16] instead of the expression given above. The normalization is done in such a way that the signal has unit power over infinite bandwidth [10]. The normalized PSD for the two different receiver type are then:

(A) Rx with CBOC reference code:

$$
\overline{G(f)}=\frac{C_{S}\left|H_{\mathrm{CBOC}}(f) H_{\mathrm{CBOC}} *(f)\right|}{\int_{-\infty}^{\infty} C_{S}\left|H_{\mathrm{CBOC}}(f) H_{\mathrm{CBOC}} *(f)\right| d f},
$$

(B) Rx with SinBOC reference code:

$$
\overline{G(f)}=\frac{C_{S}\left|H_{\mathrm{CBOC}}(f) H_{\mathrm{SinBOC}(1,1)} *(f)\right|}{\int_{-\infty}^{\infty} C_{S}\left|H_{\mathrm{CBOC}}(f) H_{\mathrm{SinBOC}(1,1)} *(f)\right| d f} .
$$

If we plot (10) and (11) as shown in Figure 3, there is almost no difference between the two transmitter-receiver

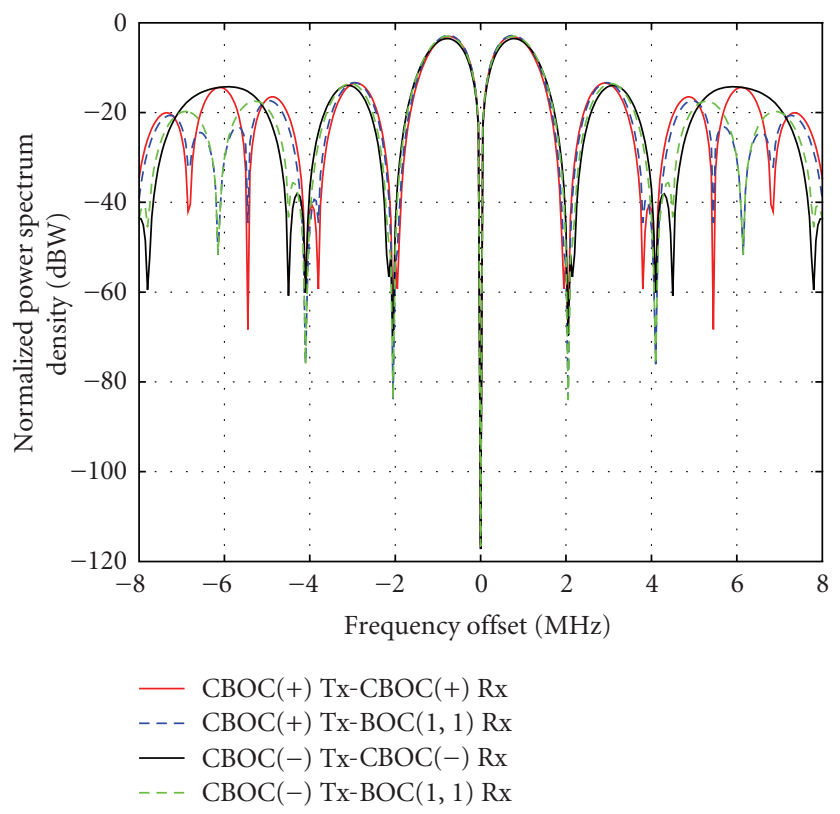

FIGURE 3: Normalized PSDs of CBOC-modulated signal receiver with $\mathrm{CBOC}$ reference code or $\operatorname{SinBOC}(1,1)$ reference code.

modulation combinations within the main lobe (between $\pm 2 \mathrm{MHz}$ ). However, outside the main lobe, difference between the power spectrums can be observed. When the BOC modulation is used in the receiver, the power spectrum from $4 f c$ to $6 f c$ has changed compared with the spectrum shown in Figure 2. The additional power is attenuated in the receiver. It means that the effect of narrowband interference will be changed. These changes on code tracking performance are analyzed in the next section.

\section{Code Tracking Error Variances}

After we derive the signal power spectrum in the receiver, the code tracking error variance shown in (2) needs to be modified accordingly. The $G_{S}(f)$ needs to be replace by the new derived $\overline{G(f)}$. Equation (2) will then become as follows: 


$$
\begin{aligned}
\left(\sigma^{2}\right)_{\text {NELP_E1 }}=B_{L} T\left(1-0.5 B_{L} T\right) \times & {\left[\frac{\int_{-\beta_{r} / 2}^{\beta_{r} / 2} G_{W}(f) \overline{G(f)} \sin ^{2}(\pi f \Delta) d f}{(2 \pi)^{2} C_{S}\left(\int_{-\beta_{r} / 2}^{\beta_{r} / 2} f \overline{G(f)} \sin (\pi f \Delta) d f\right)^{2}}\right.} \\
& \left.+\frac{\left(\int_{-\beta_{r} / 2}^{\beta_{r} / 2} G_{W}(f) \overline{G(f)} d f\right)^{2}-\left|\int_{-\beta_{r} / 2}^{\beta_{r} / 2} G_{W}(f) \overline{G(f)} e^{i 2 \pi f \Delta} d f\right|}{4(2 \pi)^{2} T C_{S}^{2}\left(\int_{-\beta_{r} / 2}^{\beta_{r} / 2} f \overline{G(f)} \sin (\pi f \Delta) d f \int_{-\beta_{r} / 2}^{\beta_{r} / 2} f \overline{G(f)} \cos (\pi f \Delta) d f\right)^{2}}\right] .
\end{aligned}
$$

The tracking error variance for a coherent early-minuslate processing (CELP) in the presence of narrowband interference then becomes

$$
\begin{aligned}
\left(\sigma^{2}\right)_{\text {CELP_E1 }}= & B_{L}\left(1-0.5 B_{L} T\right) \\
& \times\left[\frac{\int_{-\beta_{r} / 2}^{\beta_{r} / 2} G_{W}(f) \overline{G(f)} \sin ^{2}(\pi f \Delta) d f}{(2 \pi)^{2} C_{S}\left(\int_{-\beta_{r} / 2}^{\beta_{r} / 2} f \overline{G(f)} \sin (\pi f \Delta) d f\right)^{2}}\right] .
\end{aligned}
$$

Accordingly, the Cramér-Rao Lower Bound (CRLB) in the presence of narrowband interference is then:

$$
\left(\sigma^{2}\right)_{\text {LB_E1 }}=\frac{B_{L}\left(1-0.5 B_{L} T\right)}{(2 \pi)^{2} C_{S} \int_{-\beta_{r} / 2}^{\beta_{r} / 2} f^{2}\left(\overline{G(f)} / G_{W}(f)\right) d f} .
$$

Based on the theoretical prediction above, we provide numerical results in order to have some insights, which are difficult to obtain from the analytical expression. For all of the following numerical results, the E1 signal power is $-164 \mathrm{dBW}$, and the noise power density is $-204 \mathrm{dBW} / \mathrm{Hz}$, yielding a signal carrier to noise density ratio $\left(C / N_{0}\right)$ of $40 \mathrm{~dB}-\mathrm{Hz}$. The integration time used to compute the code tracking variance is $4 \mathrm{~ms}$. The front-end filter of the receiver has two different bandwidths: $4 \mathrm{MHz}$, which covers the main lobe of E1 signal power spectrum, and $14 \mathrm{MHz}$, which also includes the power allocated in the higher frequency component.

The interference used for these numerical results is the band limited Gaussian noise with $10 \mathrm{kHz}$ bandwidth and varying center frequency and power. The standard deviation of code tracking, which is the square root of code tracking variance, is analyzed as a function of the interference center frequency. It shows the effect of different placement of the interference relative to E1 signal band center. The effect of narrowband interference with varying power at fixed frequency is also studied below.

Figure 4 gives the standard deviation of code tracking error comparison between BPSK signal and CBOCmodulated signal in the presence of narrowband interference. Here, for both signals, the receiver uses the same modulation as in transmitter. As we can see that, the CBOC signal has better overall performance against the narrowband interference than the BPSK signal. This is because of the additional power on the high frequency in CBOC modulation and the narrower peak in the autocorrelation function of CBOC modulation compared with that of BPSK. For both BPSK- and CBOC-modulated signals, when the interference center frequency matches the E1 carrier, the interference does not affect the useful signal. This is because the interference is eliminated by the downconversion from Intermediate Frequency (IF) to baseband. The biggest effect of narrowband interference on CBOC signal happens at $\pm 1 f c$, $\pm 3 f c, \pm 5 f c$ away from the carrier. However, these points are the frequencies at where the interference in GPS BPSK signal has the minimum effect. This would be very useful in dual-system receiver, which is using GPS L1 and Galileo E1 signal, since the receiver can switch between tracking Galileo E1 signal and GPS L1 signal based on the detected interference frequency to avoid the big effect on the tracking performance.

The relative performance of the three considered discriminators (NELP, CELP, and CRLB) versus the interference frequency offset when the receiver utilizes the same modulation as in the transmitter is shown in Figure 5. It can be observed that the narrowband interference has the biggest effect when the interference is allocated at $1 f_{c}(1.023 \mathrm{MHz})$ away from the carrier. The effect is decreasing when the interference is moving away from the carrier. At $6 f c$, the degradation in the tracking performance is increased.

If we compare the tracking performance in presence of narrowband interference when the receiver uses difference modulation as shown in Figure 6, the $\mathrm{CBOC}(-) \mathrm{Tx}-$ $\operatorname{BOC}(1,1) \mathrm{Rx}$ combination is more robust towards the narrowband interference than other combinations. In general, if the front-end bandwidth is wide enough, using BOC modulation in the receiver has worse tracking performance than using CBOC modulation in the receiver due to the loss of signal power. However, it gives better performance if the interference is at $6 f c$ away from the carrier.

As shown in Figure 7, when the receiver front-end bandwidth is getting narrower, there is no big difference in the code tracking performance of the different transmitterreceiver modulation combinations in the presence of narrowband interference. 


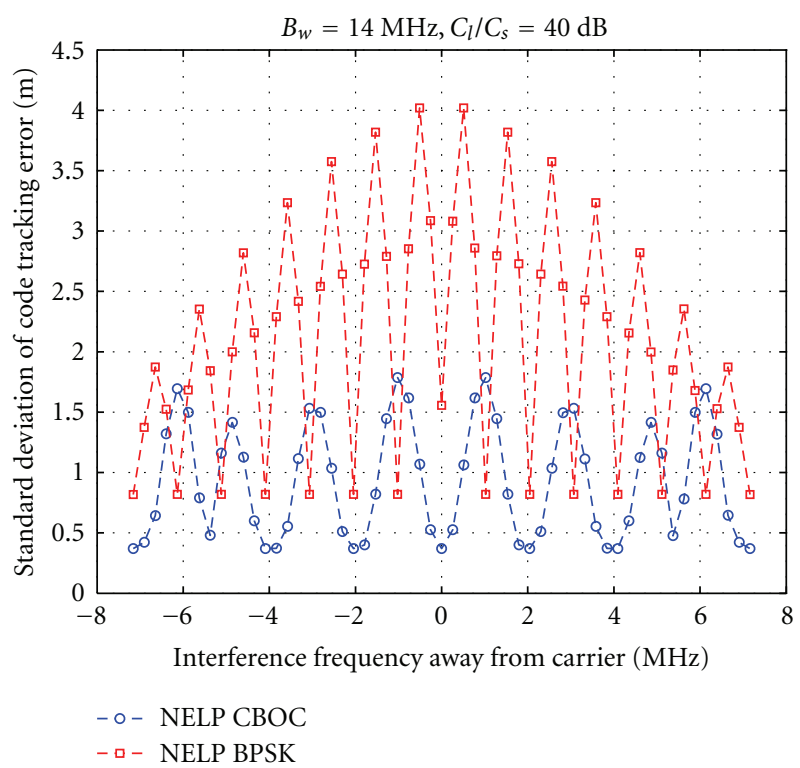

FIgURE 4: Standard deviation of code tracking error versus the center frequency of narrowband interference with CBOC and BPSK signal.

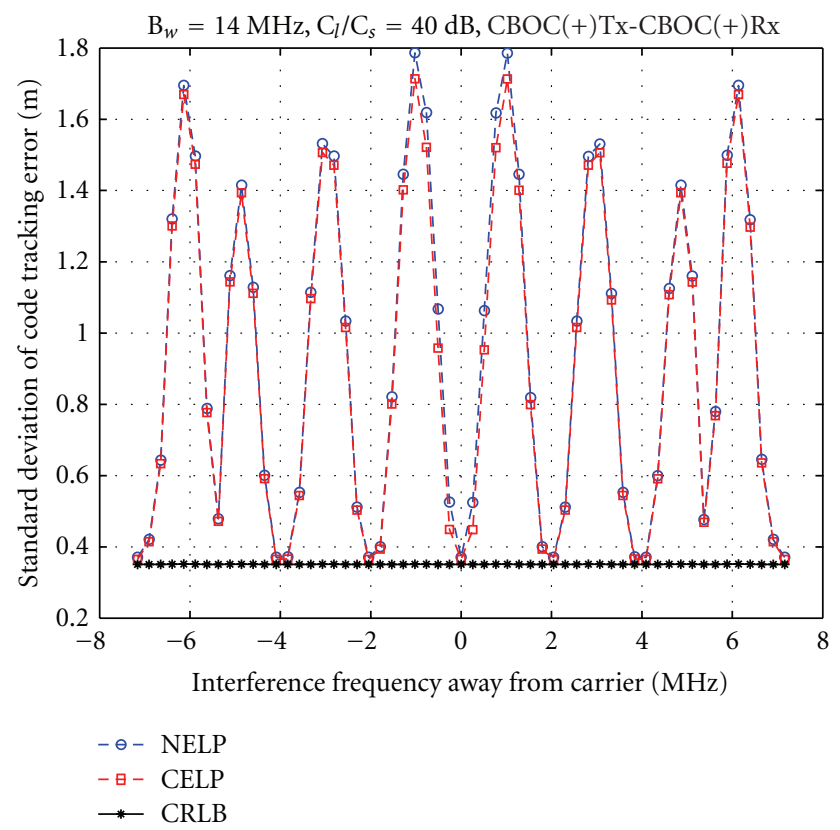

FIGURE 5: Standard deviation of code tracking error versus the center frequency of narrowband interference with CBOC signal.

Figure 8 shows the code tracking standard deviation for different interference power when the interference is placed at $1 f c$ away from the carrier. The $\mathrm{CBOC}(-)$ modulation in the transmitter with $\mathrm{CBOC}(-)$ modulation in the receiver again shows the best resistance against the narrowband interference.

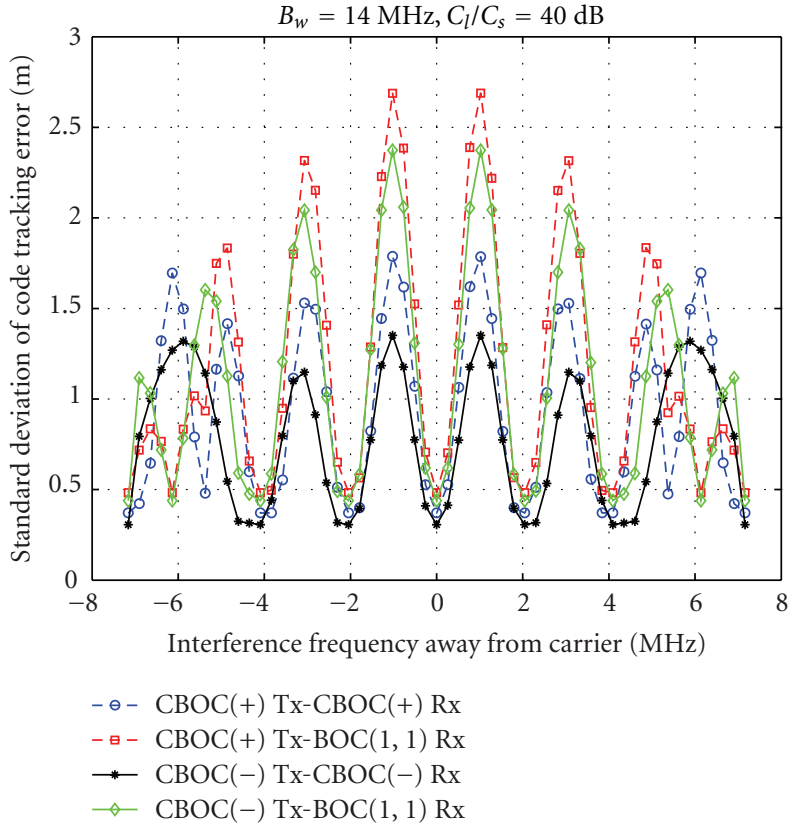

FIGURE 6: Standard deviation of code tracking error versus narrowband interference center offset for different Tx-Rx modulation combinations for $14 \mathrm{MHz}$ front-end bandwidth.

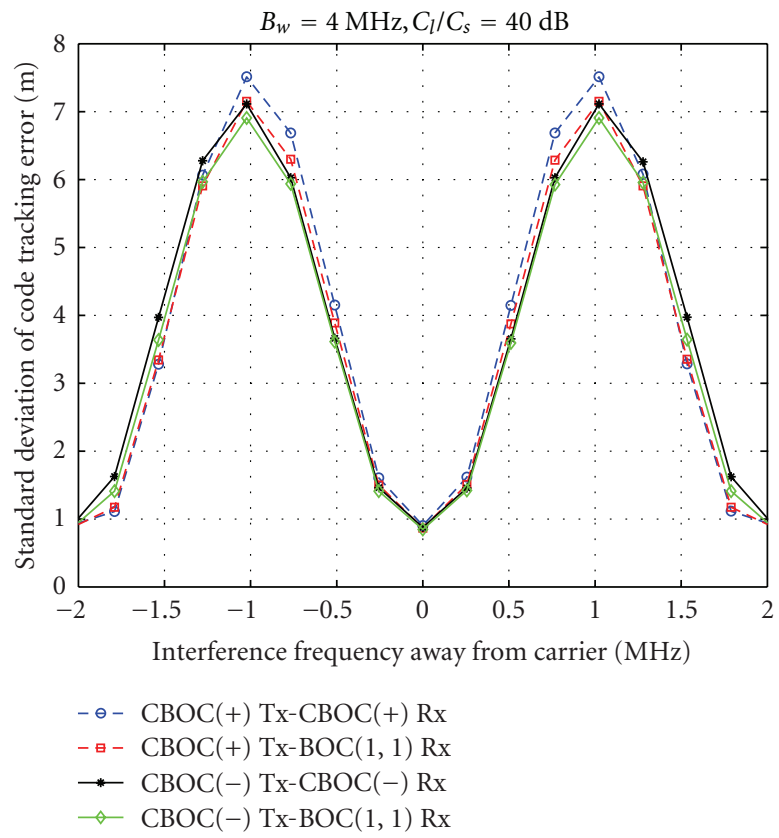

Figure 7: Standard deviation of code tracking error versus narrowband interference center offset for different Tx-Rx modulation combinations for $4 \mathrm{MHz}$ front-end bandwidth.

For the $-105 \mathrm{dBW}$ interference power, which is $60 \mathrm{~dB}$ higher than the signal, the $\mathrm{CBOC}(-)-\mathrm{CBOC}(-)$ combination has up to 12 meters less error than the $\mathrm{CBOC}(+)$ $\operatorname{BOC}(1,1)$ combination. On the other hand, if the narrowband interference is located at $6 f c$ away from the carrier, the $\mathrm{CBOC}$ modulation in the transmitter with BOC modulation 


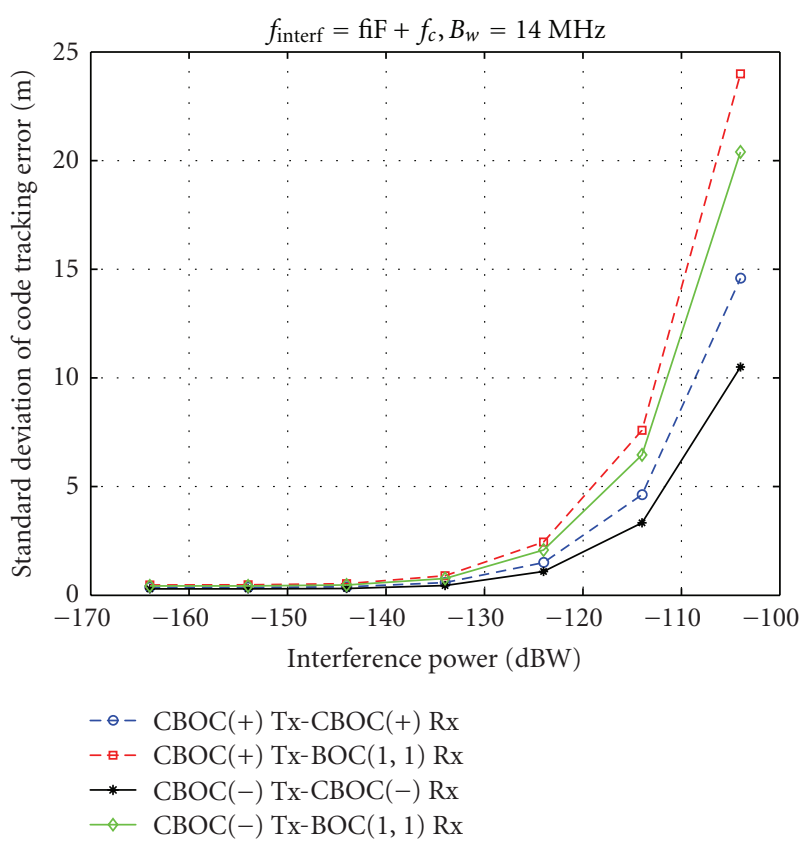

FIGURE 8: Standard deviation of code tracking error versus interference power when the interference is located at $1 f c$ away from the carrier.

in the receiver has big advantage that the code tracking performance does not degrade with the incensement of interference power as shown in Figure 9.

\section{Simulation Setup}

In order to validate the analytical expression derived in the previous section, the effect of narrowband interference is also evaluated in a link-level simulator [12]. This link-level simulator is an open source Galileo E1 signal Simulink simulator build at Department of Communications Engineering at Tampere University of Technology. The block diagram of this simulator is shown in Figure 10.

The transmitter block is implemented based on CBOC modulation, including primary code and secondary code, in accordance with the latest Galileo OS SIS ICD [13]. The transmitter consists of two channels, E1B and E1C. $\mathrm{E} 1 \mathrm{~B}$ is $\mathrm{CBOC}(+)$-modulated signal with navigation data and $\mathrm{E} 1 \mathrm{C}$ is $\mathrm{CBOC}(-)$-modulated signal with a predefined bit sequence of CS25 (i.e., pilot channel). The E1 signal is formed as the difference between those two signals. The signal at the output of the transmitter is at IF. The channel generates multipath and complex Additive White Gaussian Noise (AWGN) according to user-defined $C / N_{0}$. In this paper, we only consider single-path scenarios in order to focus on the narrowband interference effects. The receiver's front-end filter is a Chebyshev type I filter. The tracking is implemented separately for E1B channel and E1C channel. The reference code can be either CBOC-modulated (i.e., $\mathrm{CBOC}(+)$ for $\mathrm{E} 1 \mathrm{~B}$ channel and $\mathrm{CBOC}(-)$ for $\mathrm{E} 1 \mathrm{C}$ channel), or SinBOC $(1,1)$-modulated code for both E1B and E1C channels. The synchronization in the receiver is done

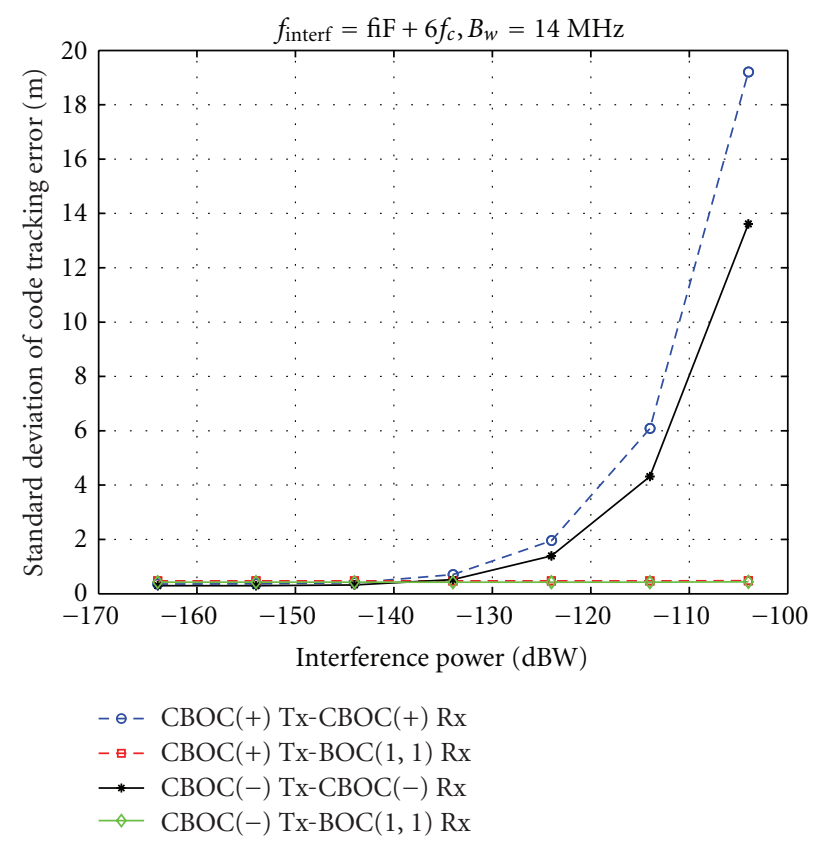

FIGURE 9: Standard deviation of code tracking error versus interference power when the interference is located at $6 f c$ away from the carrier.

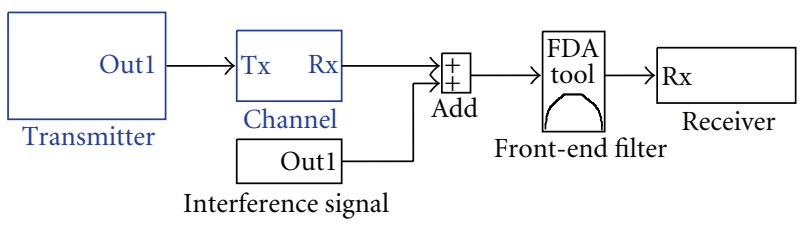

Figure 10: Galileo E1 Tx-Rx chain Simulink simulator.

TABLE 1: Simulation parameters.

\begin{tabular}{lc}
\hline Simulation parameters & Value \\
\hline Sampling frequency $(\mathrm{MHz})$ & 26 \\
Front-end bandwidth (double-sided) $(\mathrm{MHz})$ & $4 / 13$ \\
$B_{L}(\mathrm{~Hz})$ & 1 \\
Integration time $T(\mathrm{~ms})$ & 4 \\
Desired signal $C_{S} / N_{0}(\mathrm{~dB}-\mathrm{Hz})$ & 45 \\
Interference signal $C_{1} / N_{0}$ & 85 \\
Interference signal $\mathrm{BW}(\mathrm{kHz})$ & 10 \\
\hline
\end{tabular}

based on a Delay Lock Loop (DLL). In the discriminator, the Narrow Correlator (NCORR) [17] is used. The equivalent rectangular bandwidth of the code tracking loop is set to $1 \mathrm{~Hz}$ (double-sided).

Power levels of the desired signal and thermal noise were set to produce a signal with $C / \mathrm{N}_{0}$ of $45 \mathrm{~dB}-\mathrm{Hz}$. The narrowband interference is generated as that the white Gaussian noise passes through a bandpass filter (see Figure 11). The center frequency of the bandpass filter defines the interference center frequency. The simulation parameters are summarized in Table 1. 


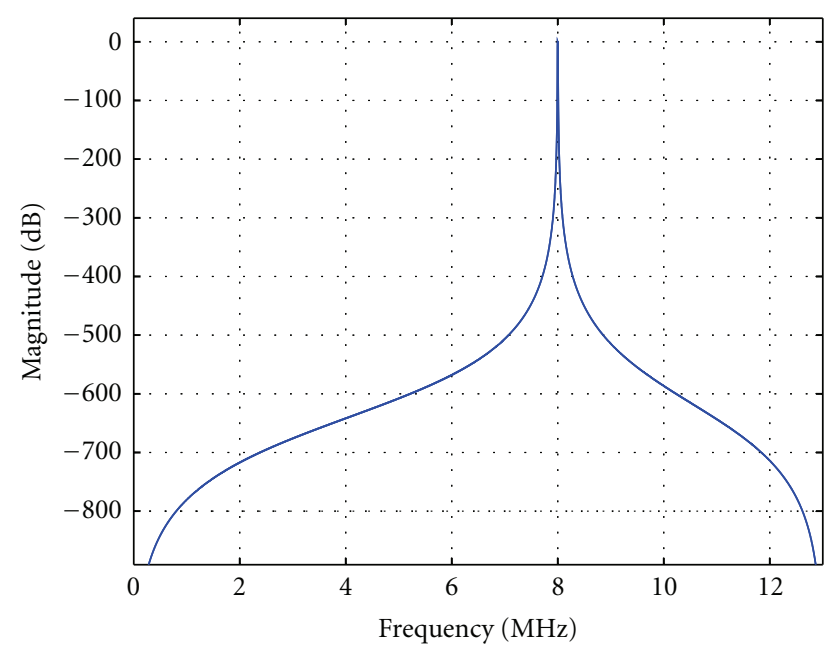

Figure 11: Example of bandpass filter use for generating narrowband interference.

\section{Simulation Results}

In this section, the simulation results of code tracking performance in the presence of narrowband interference are presented along with the discussion. The performance criteria are based on standard deviation of code tracking error, which is obtained from the Simulink model. One thing need to be mentioned here is that the results from Simulink simulation cannot be directly compared with the theoretical results. It is because the simulator tracks the $\mathrm{CBOC}(+)$ and $\mathrm{CBOC}(-)$ signals at the same time, and the code tracking output is the average of tracking output from both signals.

Figure 12 shows the standard deviation of code tracking error for different interference location. It can be observed that the biggest effect happens at $\pm f c$ away from the carrier for both receiver types. The CBOC receiver has a little better performance than SinBOC receiver against the narrowband interference within the front-end bandwidth. This is because of the narrower peak in the CBOC autocorrelation function than that in the SinBOC correlation function. The same simulations have also been done with wider front-end bandwidth as shown in Figure 13. Compared with the results in Figure 12, the tracking error is smaller regardless of receiver type. The difference between the code tracking with $\mathrm{CBOC}$ and SinBOC receiver is bigger, because the additional power is located at high frequency.

Figures 14 and 15 show the code tracking performance for the incensement of interference power when the interference is located at $1 f_{c}$ and $6 f_{c}$ away from the carrier, respectively. As can be seen, for both $\mathrm{CBOC}$ receiver and SinBOC receiver, when the interference is placed at $1 f c$, the code tracking performance is getting worse with the incensement of interference power and $\mathrm{CBOC}$ receiver is more robust towards the interference when the interference to signal power ratio is very high. This is consistent with the results shown in Figure 8. When the interference is located at $6 f c$ away from the carrier, the receiver which uses SinBOC modulation has much better code tracking performance than

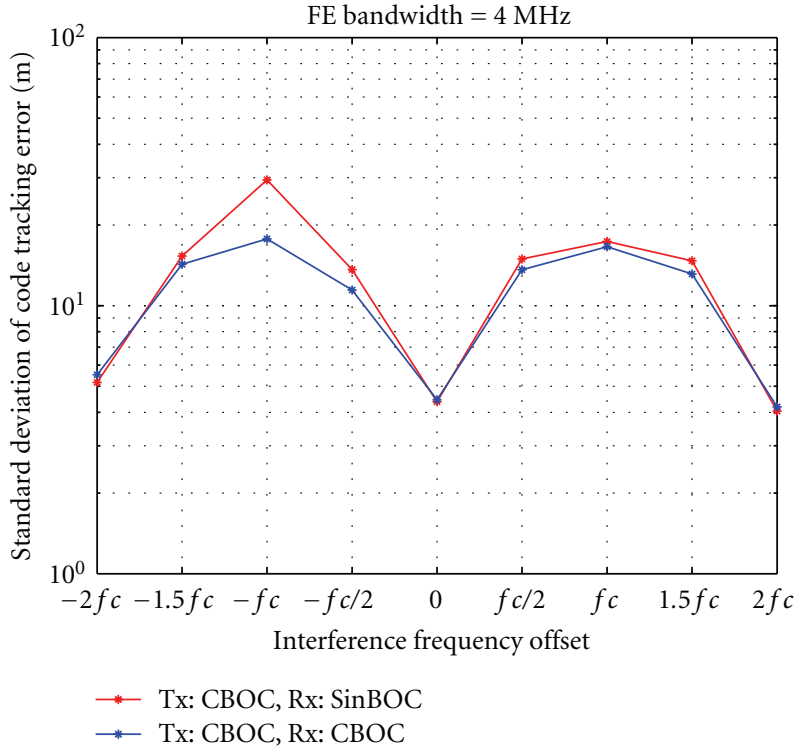

FIGURE 12: Code tracking error versus interference frequency offset related to carrier when front-end bandwidth is $4 \mathrm{MHz}$.

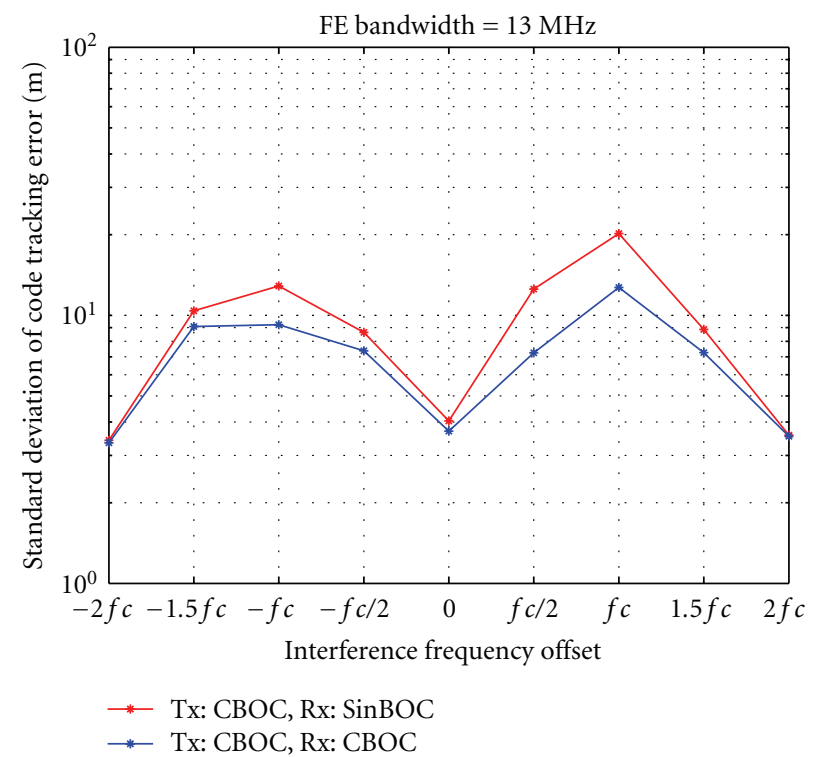

FIGURE 13: Code tracking error versus interference frequency offset related to carrier when front-end bandwidth is $13 \mathrm{MHz}$.

the receiver uses CBOC modulation. This is because that there is a null in the PSD of SinBOC-modulated signal at $6 f_{c}$ from carrier frequency. The interference will not affect the useful signal.

\section{Conclusions}

This paper has evaluated the robustness of CBOC signal towards the narrowband interference, taking into count the impact of modulation type in receiver. This paper has first overviewed the analytical model for tracking GPS BPSK signal in the presence of narrowband interference. Then 


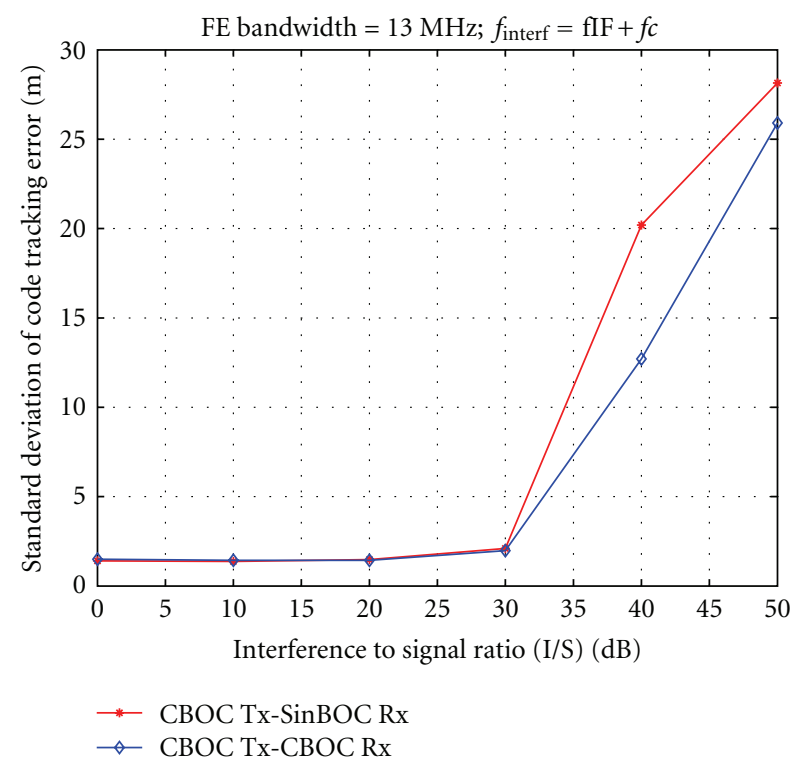

FIGURE 14: Code tracking error versus interference to signal power ratio when the interference is located at $1 f c$ away from the carrier.

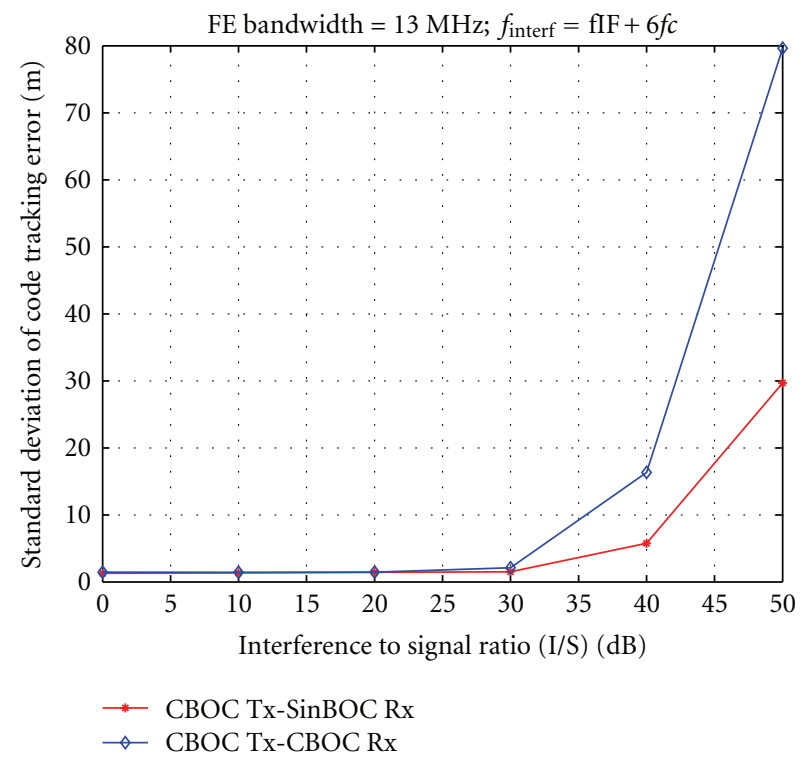

FIGURE 15: Code tracking error versus interference to signal power ratio when the interference is located at $6 f c$ away from the carrier.

the difference between the BPSK and CBOC modulation has been discussed and the impact of the modulation type in the receiver is analyzed theoretically. The analytical model for code tracking of Galileo CBOC signal in the presence of narrowband interference is presented according to the discussion of modulation type impact. The results are shown based on both derived mathematical equation and simulation in a link-level Simulink simulator.

The results obtained from the theoretical expression show that CBOC signal is more robust than the GPS BPSK signal towards the narrowband interference. For CBOC signal, regardless of the modulation type in the receiver, the narrowband interference has little effect on the carrier frequency and has the most effect when the interference is placed at one chip rate away from the carrier. The modulation used in the receiver has impact on the code tracking performance in the presence of narrowband interference of CBOC signal. In general, the $\mathrm{CBOC}$ receiver has better performance against the narrowband interference than the $\mathrm{SinBOC}$ receiver. However, if the interference is located at $6 f c$ away from the carrier, the $\mathrm{CBOC}$ receive does not give any benefit against strong interference.

For future work, the theoretical and simulation results presented here regarding the performance of E1 CBOC signal in the presence of narrowband interference will be confirmed in a hardware setup.

\section{Acknowledgments}

The research leading to these results has received funding from the European Union's Seven Framework Programme (FP7/2007-2013) under the Grant Agreement n227890 (GRAMMAR project) and from Academy of Finland, which are gratefully acknowledged. The authors would also like to thank Nokia Foundation and Tekniikan edistämissäätiö (TES) for their support.

\section{References}

[1] B. Motella, S. Savasta, D. Margaria, and F. Dovis, "A method to assess robustness of GPS C/A code in presence of CW interferences," Hindawi International Journal of Navigation and Observation, vol. 2010, Article ID 294525, 8 pages, 2010.

[2] E. D. Kaplan and C. Hegarty, Understanding GPS: Principles and Applications, Artech House Publishers, 2nd edition, 2005.

[3] F. Klinker and O. B. M. Piestersem, Interference of GPS signal, Influence of Licensed Transmitter on GPS signal Quality in the Netherlands' Airspace, National Aerospace Laboratory, 2000.

[4] J. W. Betz, "Effect of narrowband interference on GPS code tracking accuracy," in Proceedings of the 2000 National Technical Meeting of The Institute of Navigation, pp. 16-27, Anaheim, Calif, USA, January 2000.

[5] J. W. Betz, "Effect of partial-band interference on receiver estimation of C/N0: theory," in Proceedings of the National Technical Meeting of The Institute of Navigation, pp. 817-828, Long Beach, Calif, USA, January 2001.

[6] K. R. Kolodziejski and J. W. Betz, "Effect of non-white gaussian interference on GPS code tracking accuracy," The MITRE Corporation Technical Report MTR99B21R1, 1999.

[7] H. Chris, T. Michael, and L. Young, "Simplified techniques for analyzing the effects of non-white interference on GPS receivers," in Proceedings of the 15th International Technical Meeting of the Satellite Division of The Institute of Navigation (ION GPS '02), pp. 620-629, Portland, Ore, USA, September 2002.

[8] J. A. Avila-Rodriguez, S. Wallner, and G.W. Hein, "CBOCan implementation of MBOC," in Proceedings of the 1st CNES Workshop on Galileo Signals and Signal Processing, Tolouse, France, October 2006.

[9] G. W. Hein, J. A. Avila-Rodriguez, S. Wallner et al., "MBOC: the new optimized spreading modulation recommended for 
GALILEO L1 OS and GPS L1C," in Proceedings of the IEEE/ION Position, Location, and Navigation Symposium, pp. 883-892, April 2006.

[10] E. S. Lohan, "Analytical performance of CBOC-modulated Galileo E1 signal using sine $\mathrm{BOC}(1,1)$ receiver for massmarket applications," in Proceedings of the IEEE PLANS, Position Location and Navigation Symposium, pp. 245-253, Indian Wells, Calif, USA, May 2010.

[11] J. Zhang and E. S. Lohan, "Multi-correlator structures for tracking Galileo signals with $\mathrm{CBOC}$ and $\operatorname{SinBOC}(1,1)$ reference receivers and limited front-end bandwidths," in Proceedings of the 7th Workshop on Positioning, Navigation and Communication (WPNC '10), pp. 179-186, Dresden, Germany, March 2010.

[12] Simulink open-source software for Galileo E1 signals, Tampere University of Technology, http://www.cs. tut.fi/tlt/pos/Software.htm.

[13] Galileo Open Service Signal In Space Interface Control Document, (SIS-ICD08), http://www.gsa.europa.eu/go/galileo /os-sis-icd/galileo-open-service-signal-in-space-interface -control-document.

[14] J. W. Betz, "The Offset Carrier Modulation for GPS modernization," in Proceedings of the National Technical Meeting of The Institute of Navigation, pp. 639-648, 1999.

[15] B. A. Siddiqui, J. Zhang, M. Z. H. Bhuiyan, and E. S. Lohan, "Joint Data-Pilot acquisition and tracking of Galileo e 1 Open Service signal," in Proceedings of the Ubiquitous Positioning Indoor Navigation and Location Based Service (UPINLBS '10), pp. 1-7, October 2010.

[16] G. Artaud, L. Ries, J. Dantepal, J. Issler, T. Grelier, and A. Delatour, "CBOC performances using software receiver," in Proceedings of the 2nd Workshop on GNSS Signals \& Signal Processing (GNSS SIGNALS '07), Nordwijk, Netherlands, October 2007.

[17] J. W. Betz and K. R. Kolodziejski, "Extended theory of earlylate code tracking for a bandlimited GPS receiver," Journal of the Institute of Navigation, vol. 47, no. 3, pp. 211-226, 2000. 

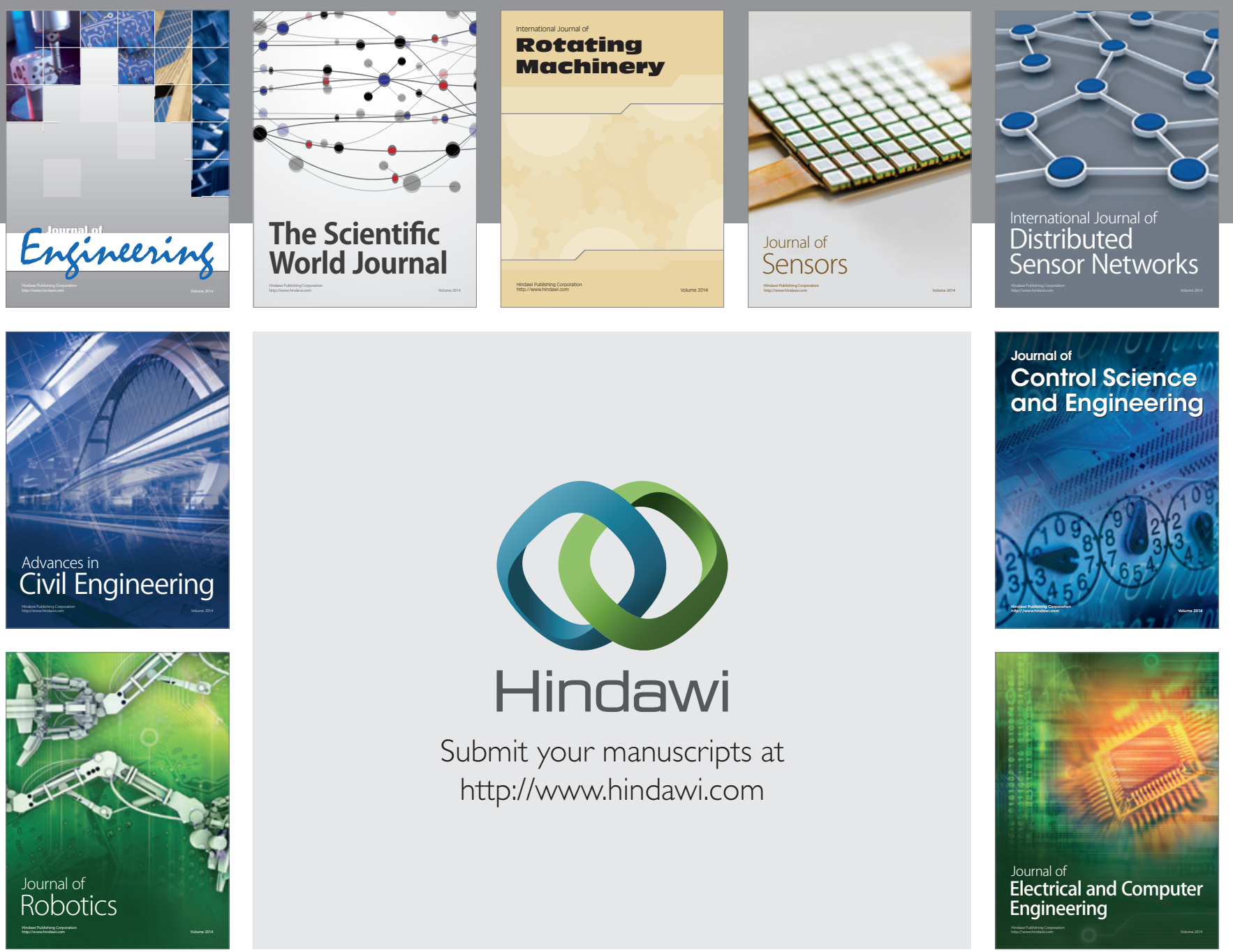

Submit your manuscripts at

http://www.hindawi.com
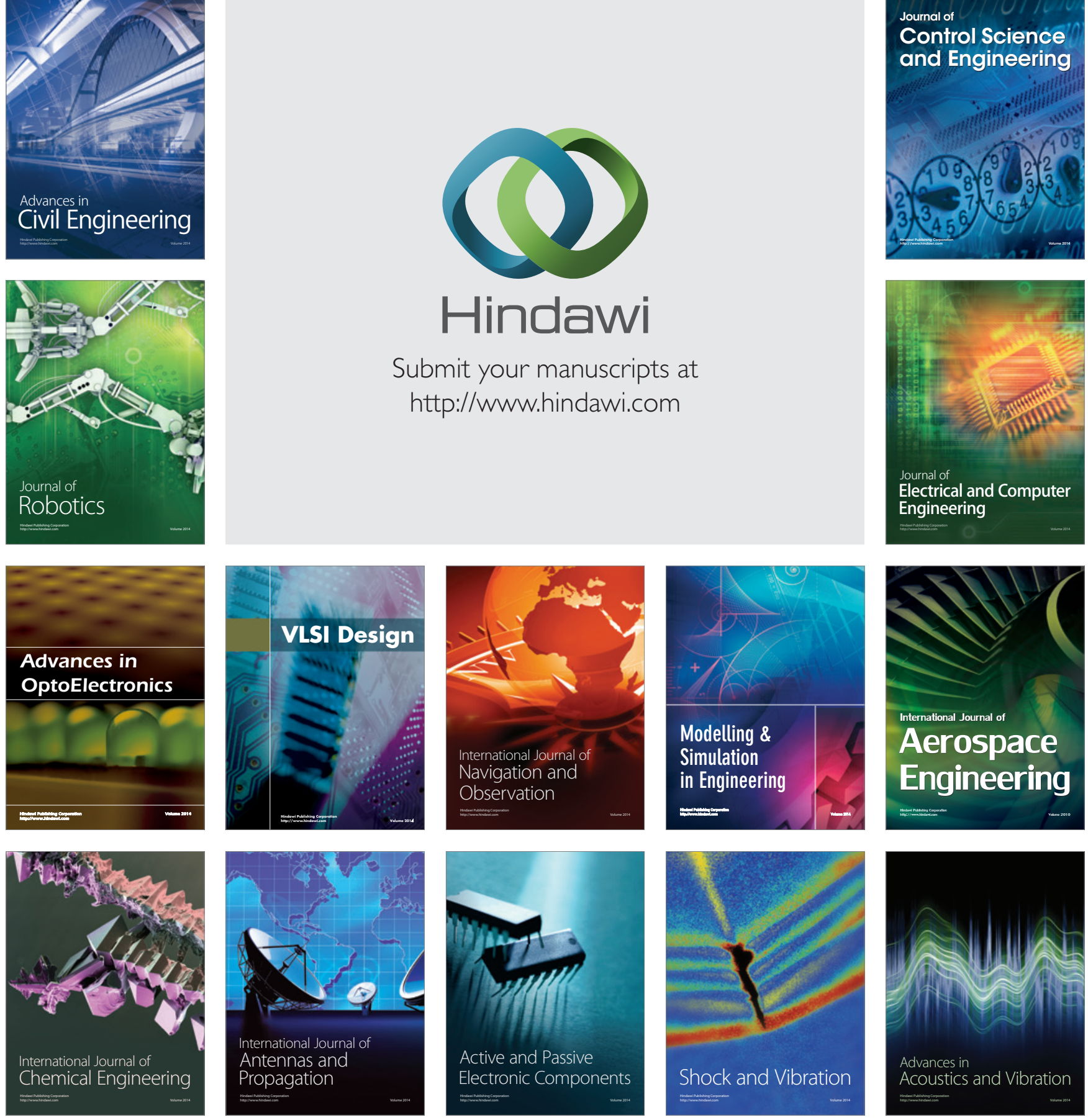